\title{
Instability of the Determinants of Birth Intervals in Ahvaz-Iran: A Structural Change Modelling Approach
}

\author{
Abdolrahman Rasekh* and Hadi Imami \\ Shahid Chamran University
}

\begin{abstract}
Birth interval is one of the major determinants of the fertility rate. In this paper structural change approach is used to study the instabilities in the effects of different socio-economic factors on birth intervals of children with respect to couple's years of marriage factor in Ahvaz-Iran. A class of M-fluctuation tests for parameter instability is used and based on these tests different evidences of instability including the years of change and factors which changed are estimated and identified. The data analysed come from a sample of women referred to "Health and Medical Centres" during October and November 2002.
\end{abstract}

Keywords. Birth intervals; change point; fluctuation test; mortality.

MSC 2010: 62J05.

\section{Introduction}

Fertility, as one of the most important factors in determining variations in populations, has high special emphasis in population research. To study fertility behaviour of women event histories such as birth, pregnancy and marriage have been used by social scientists. Analysis of sequence in the childbearing process could provide a more comprehensive picture of the dynamics of fertility transition. Birth history analysis undoubtedly provides

* Corresponding author 
useful information considering reproduction and family formation. The factors affecting fertility may have varying effects on child spacing. Thus birth intervals (BI) may reveal some insight about their reproduction patterns.

Differences in exposure to the risk of pregnancy and differences in the length of time between births when women are exposed may contribute to differentials in childbearing levels (Trussel et al., 1985). Whatever the cause, the length of birth intervals may vary from one group of women to another.

Differences in birth intervals length are explained by varying breastfeeding patterns, frequency of intercourse, contraceptive use, incidence of abortion and fecundity. Other factors such as women roles and status and the value of the children may also affect the birth intervals. Undoubtly the socio-economic, demographic, health and cultural background of a country, consequently that of women, influence the birth intervals.

Age at marriage and women's education are the most widely analyzed determinants of birth intervals. The first one is found to have a substantial effect on birth intervals. If on couples marry at a very young age, couple's decision on the number of children, contraceptives, and the like may be formed at less mature age, consequently affecting the birth intervals (Bumpass et al., 1987). In fertility process for younger women West (1987) found that the first birth is an important determinant at the transition from parity one to parity two. Abdel Aziz (1983) concluded that the later placecountry-regionJordan girl marries, the swifter she will bear her first child.

Women's Education is considered to be an important variable in birth intervals analysis. However in another study Nair (1996) did not found any significant effect in terms of the education of women on the birth intervals. In addition, the female education was found to be an insignificant determinant of the risk of pregnancy in Malaysia. Nevertheless, the male education and occupation were found to be significant factors on birth intervals in Indonesia and the Philippines (Trussel et al., 1985).

Economic status such as type of individual's home status and kinship status may have an influence on birth intervals. A couple's decision on the timing of the first, second or the third baby may depend on traditional and cultural practices. Rashid Mahan (1997) studied effects of lactation, contraception and other factors on birth intervals in Bangladesh. Suwal (2001) considered socio-cultural dynamics of birth intervals in Nepal. She founds that the first births are much more important than the subsequent births in Nepalese society.

In Iran, among different works one can refer to Rasekh and Momtaz 
(2007) who studied the determinants of birth intervals in Ahvaz-Iran using a graphical modeling approach and Amani et al. (2011) who studied mortality rate using change points analysis.

Using structural change approach this paper intends to explore the changes of effect of socio-economic, demographic, cultural differentials, and healthrelated factors on birth intervals of children along with the couple's years of marriage in Ahvaz-Iran. In our approach we investigate instability in multiple linear regression relationship between birth intervals as dependent variables and socio-economic factors as explanatory variables along with the couple's years of marriage. A class of recent M-fluctuation test for parameter instability is used. Evidence for structural change, the change years and factors which their effects changed over these years are identified and estimated.

The reminder of this paper is organised as follows: in Section 2 we give a brief review of the structural change models with segmented regression. In Section 3 we introduce the collected data set and in Section 4 structural change is used to analyse the 1st and 2nd birth intervals and to derive different evidences of instability including the years of change and factors which changed.

\section{Structural Change Models}

In many fields of research scientists are interested to learn when and how the structure of the data generating mechanism underlying a set of observations changed. Usually, it is known with respect to which quantity the structural change might occur, e.g., over time or with the increase of a certain risk factor. One of the simplest examples for such a structural change is a time series whose mean changes at a single Breakpoint.

In parametric models, structural change is typically described by parameter instability. If this instability is ignored, parameter estimates are generally not meaningful, inference is severely biased and forecasts lose accuracy.

Fields of application of structural change are manifold and include, for example, finance (see e.g., Androu and Ghysels, 2002), economics (see e.g., Stock and Watson, 1996), political and social sciences (see e.g., Cooper, et al., 2003), or biostatistics and medical statistics (see e.g., Muggeo, 2003).

Starting from the recursive CUSUM test of Brown, et al. (1975) a large variety of tests for structural change has been suggested. Two frameworks for testing structural change can be distinguished: (i) F-statistic (Andrews, 
1993; Andrews and Ploberger, 1994) that is designed for a specific alternative and (ii) generalized fluctuation tests (Kuan and Hornik, 1995) that do not assume a particular pattern of deviation from the null hypothesis.

For models estimated by maximum likelihood, Hjort and Koning (2002) used a similar approach as generalized fluctuation method. Zeleis and Hornik (2003) generalized these frameworks based on partial sum fluctuation processes of M-estimation scores. In this paper to investigate the changes in birth intervals data structure, we have used the M-fluctuation test. Such tests are considered not only significance tests but also explorative tools.

\subsection{Theory}

In structural change problems, it is of interest to test the hypothesis that the parameters of a certain model remain constant over all observations against the alternative that they change over "ordered external variable".

Suppose that $Y_{i}(i=1, \ldots, n)$ is $\mathrm{n}$ possibly vector-valued independent observations ordered with respect to an external variable (usually time) and distributed according to some distribution $\mathrm{F}$ with $\mathrm{k}$-dimensional vector parameter $\theta_{i}$ as

$$
Y_{i} \sim F\left(\theta_{i}\right) \quad i=1, \ldots, n .
$$

The null hypothesis is that the parameters are constant

$$
H_{0}: \theta_{i}=\theta_{0} \quad i=1, \ldots, n,
$$

against the alternative that (at least one component of) $\theta_{i}$ varies over "time". The idea of the tests is to estimate the vector parameter once for all $\mathrm{n}$ observations based on an M-estimation score function $\psi(\cdot, \cdot)$ which has zero expectation at the true parameters and to use the (scaled) cumulative sum process of these scores to detect instabilities in the parameters

$$
\sum_{i=1}^{n} \psi\left(Y_{i}, \hat{\theta}\right)=0
$$

Also $\psi(\cdot, \cdot)$ could be the partial derivative of the residual sum of squares or the Log-Likelihood, yielding the ordinary least squares or maximumlikelihood estimators $\hat{\theta}$, respectively. Under parameter stability, the scores $\psi\left(Y_{i}, \hat{\theta}\right)$ have zero mean but under the alternative there will be systematic deviations 
from zero which can be captured using the cumulative sum process of the scores (see Zeileis and Hornik, 2003):

$$
W_{n}(t, \hat{\theta})=n^{-\frac{1}{2}} \sum_{i=1}^{[n t]} \psi\left(Y_{i}, \hat{\theta}\right) .
$$

In which $[n t]$ is the integer part of $n t$. Functional central limit theorem (FCLT) implies that the process $W_{n}(\cdot, \cdot)$ under null hypothesis converges to a process $Z(\cdot)$, that is a Gaussian process with zero mean and covariance function

$$
\operatorname{cov}[Z(t), Z(s)]=\min (t, s) J,
$$

where $J$ is the (asymptotic) covariance matrix of the scores and can be estimated by

$$
\hat{J}=n^{-1} \sum_{i=1}^{n} \psi\left(Y_{i}, \hat{\theta}\right) \psi\left(Y_{i}, \hat{\theta}\right)^{\mathrm{T}} .
$$

Finally, given a consistent covariance matrix $\hat{J}$, the empirical fluctuation process can be denoted by:

$$
\text { efp }(t)=\hat{J}^{-\frac{1}{2}} W_{n}(t, \hat{\theta}) .
$$

Under the null hypothesis of $(2)$ and in the intervals $[0,1]$, the empirical fluctuation process efp $(\cdot)$ converges to a k-dimensional Brownian bridge $W^{0}(t)$ which can also be written as $W^{0}(t)=W(t)-t W(1)$, where $W(\cdot)$ is a standard k-dimensional Brownian motion. Under the alternative, the fluctuation should generally be increased and the process should typically exhibit peaks at the times changes in $\theta_{i}$ occur.

\subsection{Fluctuation Tests}

Following Zeileis and Hornik (2003), given a finite sample of size $n$ as in model (1), an empirical fluctuation process is an $n \times k$ array $\left(e f p_{j}(i / n)\right)_{i, j}$ where $i=1, \ldots, n, j=1, \cdots, k$ and $\operatorname{efp}_{j}(\cdot)$ is the $j$ th component of efp(.). To aggregate this empirical process to a scalar test statistic several suitable functional of the form $\lambda\left(e f p_{j}(i / n)\right)$ are conceivable. The functional $\lambda$ can usually be split into two components: $\lambda_{\text {time }}$ which aggregates over time and $\lambda_{\text {comp }}$ which aggregates over the $k$ components of process.

Common choices for $\lambda_{\text {time }}$ are the absolute maximum, the mean or the range. Typical functionals $\lambda_{\text {comp }}$ include the maximum norm (or $L_{\infty}$ norm, 
denoted as $\|\cdot\|_{\infty}$ ) or the squared Euclidean norm (or $L_{2}$ norm, denoted as $\|\cdot\|_{2}^{2}$ ). One can refer to Hjort and Koning (2002) for more details.

The test statistics in this paper are all of the form

$$
\lambda_{\text {time }}\left\{\frac{\lambda_{\text {comp }}(e f p(t))}{d(t)}\right\}
$$

where $d(\cdot)$ is a weighting function and are as follows:

Double Max Test: the only class of test statistics which allows for both identification of the component $j$ as well as the timing $i / n$ of a potential structural instability is when the maximum is used for aggregating over both time and components, i.e.,

$$
\max _{\mathrm{i}=1, \ldots, \mathrm{n}} \max _{\mathrm{j}=1, \ldots, \mathrm{k}}\left|\frac{e f p_{j}\left(\frac{i}{n}\right)}{d\left(\frac{i}{n}\right)}\right| .
$$

If this test statistic crosses some absolute critical value $c$, it can be regarded as violating the hypothesis of stability (Mazanec and Strasser, 2000).

SupLM Test: for testing a single abrupt change of unknown timing a method is to compute some test statistic for all potential change points in an interval $\Pi$ and reject if their maximum is too large (Andrews, 1993). By applying the squared Euclidean norm of the empirical fluctuation process weighted by its variance the SupLM statistic is

$$
\sup _{t \in \Pi} \frac{\|\operatorname{efp}(t)\|_{2}^{2}}{t(1-t)}
$$

More precisely, $\lambda_{\text {comp }}$ and $\lambda_{\text {time }}$ are $\|\cdot\|_{2}^{2}$ and $\sup _{t \in \Pi}$, respectively, and $d(t)=$ $t(1-t)$. Hence the limiting distribution is given by

$$
\sup _{t \in \Pi}\left\{(t(t-1)\}^{-1}\left\|W^{0}(t)\right\|_{2}^{2} .\right.
$$

By method of Hansen (1997) the critical values can be computed. The aveLM and expLM can be derived analogously, with the same $\lambda_{\text {comp }}$ and dand replacing only $\lambda_{\text {time }}$ time by the average and the exp functional, respectively. The aveF and expF test have certain optimality properties (Andrews and Ploberger, 1994). 


\subsection{Segment Regression and Dating Structural Changes}

Based on the above mentioned tests if there is significant evidence for structural change in model, a natural approach is to estimate the breakpoints and restructure the model by these breakpoints. The estimation of the corresponding breakpoints is also called dating of structural changes. In several statistical disciplines and communities, methods have been developed that address this problem from different points of view. Assuming that there are $\mathrm{m}$ breakpoints, thus there are $\mathrm{m}+1$ segments in which the regression coefficients are constant, and the model (1) can be rewritten as

$$
y_{i}=x_{i}^{\mathrm{T}} \beta_{i}+\varepsilon_{i} \quad i=i_{j-1}+1, \ldots, i_{j}, j=1, \ldots, m+1
$$

where $j$ is the segment index, $I m, n=\left\{i_{1}, \ldots, i_{m}\right\}$ denotes the set of the breakpoints (Im, $n$ is also called $m$-partition), and by convention $i_{0}=0$ and $i_{m+1}=n$. Given an $m$-partition $i_{1}, \ldots, i_{m}$ the least squares estimates for the $\beta_{j}$ can be easily obtained. The resulting minimal residual sum of squares is given by

$$
R S S\left(I_{m, n}\right)=\sum_{j=1}^{m+1} r s s\left(i_{j-1}+1, i_{j}\right),
$$

where $\operatorname{rss}\left(i_{j-1}+1, i_{j}\right)$ is the usual minimal residual sum of squares in the $j$ th segment. The problem of dating structural changes is to find the breakpoints $\hat{i}_{1}, \ldots, \hat{i}_{m}$ that minimize the objective function

$$
\left(\hat{i}_{1}, \ldots, \hat{i}_{m}\right)=\arg \min _{\left(I_{m, n}\right)} R S S\left(I_{m, n}\right)
$$

over all partitions $I_{m, n}\left(i_{1}, \ldots, i_{m}\right)$ with $i_{j}-i_{j-1} \geqslant n_{h} \geqslant k$.

To obtain global minimum in (8), Bai and Perron (2003) present a dynamic programming algorithm for structural change models in an OLS regression context. Following Bai and Perron (2003) the optimal segmentation satisfies the recursion

$$
R S S(\operatorname{Im}, n)=\min _{m n_{h} \leqslant i \leqslant n-n_{h}}[R S S(\operatorname{Im}-1, i)+r s s(i+1, n)] .
$$

Therefore, for each point $i$, if $i$ was the last breakpoint in an m-partition the "optimal previous partner" can be derived from triangular matrix of $r s s(i, j)$ with $j-i \geqslant n_{h}$. For more details of dynamic programming see Bai and Perron (2003). 
For estimating the number of breakpoints a common procedure is to consider information criteria. Yao (1988) suggests the use of Bayesian Information Criterion (BIC) defined as

$$
B I C(m)=\ln \hat{\sigma}^{2}(m)+\{(m+1) q+m+p\} \ln (n) / m
$$

where $p$ is the number of parameters which have not changed and $q$ is those changed over time and $\hat{\sigma}^{2}(m)=n^{-1} R S S\left(\hat{I}_{m, n}\right)$.

Such information criteria are often used for model selection, which in this case means selection of the number $\mathrm{m}$ of breakpoints obtained by dynamic programming. Bai and Perron (2003) argue that the AIC usually overestimates the number of breaks but BIC is a suitable selection procedure in many situations.

\section{Background and Study Data}

Examination of birth intervals and factors affecting them is a method to study the fertility behavior of a society. In addition to couple's decision, socio-economic and cultural-political factors have dominant effect on birth intervals. However, effects of these factors may have changed over recent decades when marriages have formed.

In the last 30 years, birth intervals and related parameters including fertility rate have shown considerable changes in Iranian society. In order to control the fertility rate, different official programs such as change legal marriage age, promoting contraceptive use and encouragements for fewer children for better life had begun. Occurrence of Islamic revolution and IraqIran war caused some programs to stop or even get reversed. Associating with other factors, these events had special effect on fertility rate which can be considered in demography.

In this study, we examine the changes in effects of socio-economic factors which have influence on the 1st and 2nd birth intervals along with couple's years of marriage. One aim of this study is to find years that birth intervals and corresponding factors have linear stable structure. The data set used in this study contain a sample of 1000 married women aged 18-55 years that referred to "Health and Medical Centers" in Ahvaz City the Capital of Khuzestan province in south west of Iran during October and November 2002. For the collection of data, we employed intensively trained and supervised team of female interviewers. Because of the personal nature of the survey content and 
Table 1. Description of quantitative variables

\begin{tabular}{ll}
\hline \hline \multicolumn{1}{c}{ Variable } & Scale \\
\hline 1st birth interval (FBI) (Response) & Month \\
2nd birth interval (Response) & Month \\
Parents' view about ideal number of children (IN) & Count \\
Women's view about birth interval (BI) & Month \\
Women's age at marriage (AW) & Year \\
Duration of 1st child breastfeeding (TB) & Month \\
Age at marriage & Year \\
\hline
\end{tabular}

Table 2. Description of categorical variables

\begin{tabular}{|c|c|}
\hline Variable & Categoriess \\
\hline Use of Contraceptive Method (UC) & $\begin{array}{l}\text { None } \\
\text { Natural } \\
\text { Device (ref*) }\end{array}$ \\
\hline Sex of the 1st child (sex) & $\begin{array}{l}\text { male } \\
\text { Female }\end{array}$ \\
\hline Home ownership (Home) & $\begin{array}{l}\text { Owner } \\
\text { tenant } \\
\text { Other }\end{array}$ \\
\hline Women's access to media (SW) & $\begin{array}{l}\text { Seldom (ref) } \\
\text { Sometimes } \\
\text { Always }\end{array}$ \\
\hline Women's education level (LW) & $\begin{array}{l}\text { College } \\
\text { High school } \\
\text { Secondary } \\
\text { Primary } \\
\text { None (ref) }\end{array}$ \\
\hline Wanting 2nd child (WC) & $\begin{array}{l}\text { None } \\
\text { Both (ref) }\end{array}$ \\
\hline Men's ethnicity (ME) & $\begin{array}{l}\text { Persian } \\
\text { Arab } \\
\text { Bakhtiari } \\
\text { Other (ref) }\end{array}$ \\
\hline
\end{tabular}

* Reference category

cultural situation of region, we employed female interviewer. The data consist of birth intervals as response variables, versus explanatory variables including couples' views about number of children, contraceptive use before 
1st and 2nd birth, sex of 1stchild, and others such as socioeconomic, demographic and health related variables. Brief definitions of the quantitative and categorical effective explanatory variables are given in tables 1 and 2, respectively. The collected data set is available from the 1st author upon request.

\section{Findings}

In this section, we first study the structure of the 1st and 2nd birth intervals means. By fitting multiple linear regression and using structural change approach, we investigate the changing effects of above mentioned determinant variables on birth intervals during the time of couples' marriage. The data were analyzed using the R 2.1.1 and the package strucchange 1.2-11 (Zeileis, et al., 2002). The results are given in next sections.

\subsection{The 1st Birth Interval}

By modeling the 1st birth interval as a series of approximately normal observations, we first assess the stability of both the mean and the variance of the 1 st birth interval length. The estimated mean and variance under the null hypothesis are 17.90 and 160.52 month, respectively, which are tested using Double max function given in (6). This test is performed graphically in Figure 1 by plotting the process for both parameters with a horizontal boundary

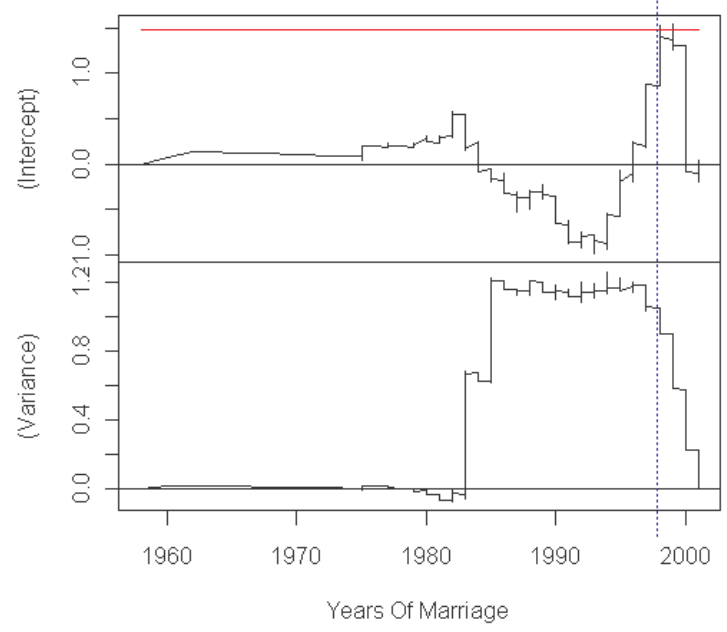

Figure 1. M-fluctuation process (Double max test) for mean and variance of 1st birth intervals 
Table 3. Test statistics and estimates of the 1st birth intervals mean with one break (1998)

\begin{tabular}{lcc}
\hline \hline & Sup LM & Double Max \\
\hline Test statistic & $13.94^{* *}$ & $1.38^{* *}$ \\
Break=(1998) & Before 1998 & After 1998 \\
Mean of the 2nd birth intervals & $17.58^{* * *}$ & $19.01^{* * *}$ \\
Std. error & 0.46 & 0.88 \\
\hline *Significant at 5\% & & \\
** significant at 1\% & & \\
$* * *$ Significant at $0.1 \%$ & & \\
\end{tabular}

for \pm the critical value at $\% 5$ level. There is only significant fluctuation in the process for mean but no boundary crossing for the variance process. It can be concluded that this instability is caused by a change in the mean of the 1 st birth intervals while no change occurs in the variance. The clear peak in the mean process conveys the information that there is single abrupt increase in the mean in 1998. Table 3 shows the estimate of 2 nd birth intervals mean changed in 1998. From this table it can be seen that the mean of 1st birth intervals has increased since 1998.

To draw valid conclusion from the effects of factors on the 1st birth interval, we first assess the stability of effects over the time of couple's marriage using the associated empirical process given in (4). The multivariate fluctuation process of Double max test shows that the fluctuation in the effects during the couple's years of marriage is very small and not significant and the process doesn't exceed their $5 \%$ level boundaries. Although the intercept of model exceed its boundary but this confirm that the mean of the 1st interval changed over couple's years of marriage (Figure 2). Thus effects of Socio- economic factors on the 1st birth interval don't reveal any change along couple's years of marriage.

Based on results from Figure 2, we fit multiple linear regressions to the 1st birth interval as response variable versus socio-economic and cultural variables. The results are given in Table 4 (it is worth to mention that we entered all available variables in the model but we report only the results of more important variables).

As we can see from the Table 4, use of contraceptive has significant effect on 1st birth interval. Comparing with those using contraceptive method (device using no contraceptive device have reverse effect on 1st birth interval. 


\subsection{The 2nd Birth Interval}

In this section, we turn our attention to the 2nd birth interval. The estimated mean and variance under null hypothesis are 48.24 and 633.02 month, respectively. The same as previous section we first assess the stability of 2 nd birth interval mean during the years of couple's marriage.

To test for structural change, we use Double max test. The process is plotted in Figure 3. The process for mean shows significant departures from the null at $5 \%$ level (indicating break points in the 2 nd birth interval),
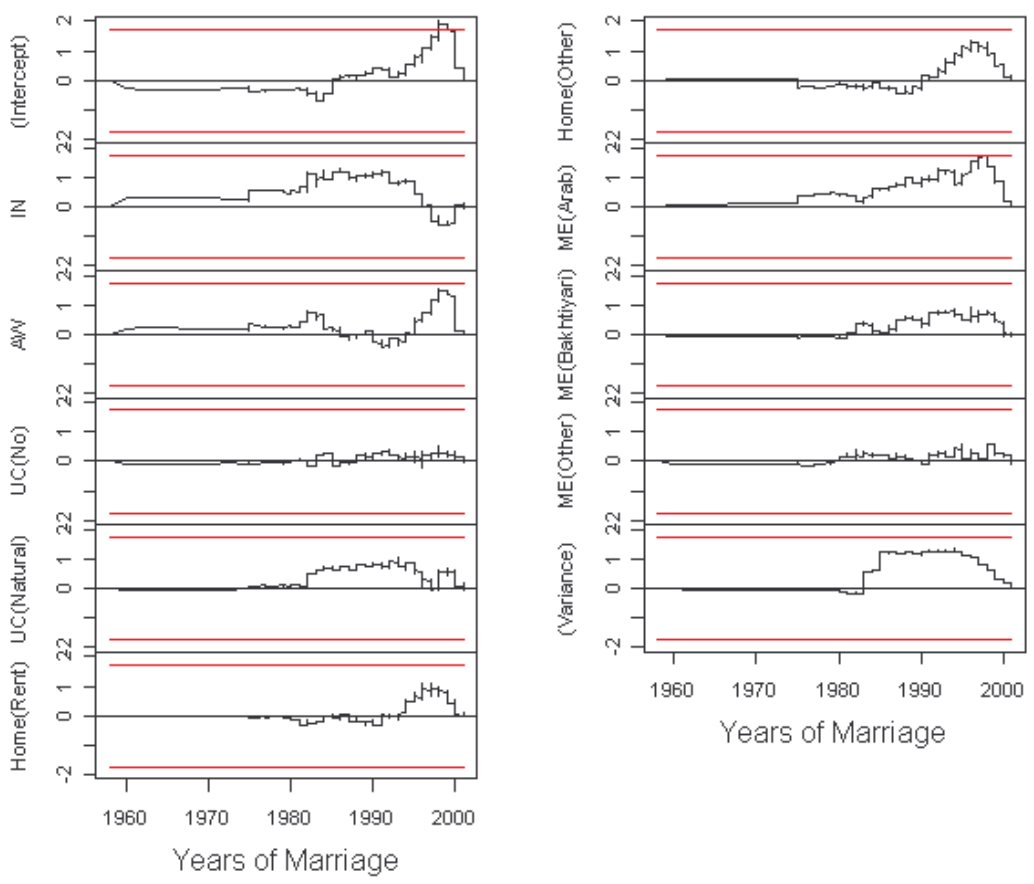

Figure 2. 10-dimensional empirical M-fluctuation process for 1st BI data with double maximum functional 
Table 4. Estimated effects of socio-economic factors on 1st BI

\begin{tabular}{lcc}
\hline \hline Variable & Estimate & Std. Error \\
\hline Constant & $28.74^{*}$ & 5.63 \\
Ideal number of children (IN) & -0.23 & 0.31 \\
Women's age at 1st marriage (AW) & $-1.03^{* *}$ & 0.10 \\
Use of Contraceptive method (UC) & & \\
$\quad$ No & $-3.76^{* *}$ & 1.07 \\
$\quad$ Natural & -1.68 & 1.27 \\
$\quad$ device (ref) & - & - \\
Home status & & \\
Tenant & -2.08 & 1.69 \\
Other & $-3.25^{*}$ & 1.37 \\
Owner (ref) & - & - \\
Men's ethnicity & & \\
Arab & -0.80 & 0.97 \\
Bakhtiyari & -0.25 & 1.41 \\
Persian & 1.46 & 1.58 \\
Other (ref) & - & - \\
\hline
\end{tabular}

*Significant at $5 \%$

** significant at $1 \%$

$* * *$ Significant at $0.1 \%$

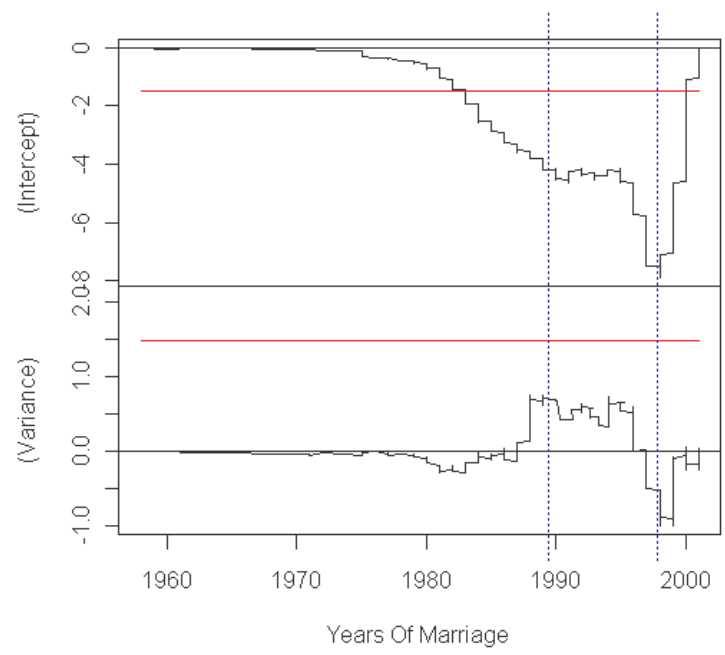

Figure 3. M-fluctuation process (Double max test) for the 2nd birth intervals 
Instability of the Determinants of Birth Intervals in Ahvaz-Iran: A Structural ...

Table 5. Test statistics and estimates of the 2nd birth intervals mean with two breaks

\begin{tabular}{lccc}
\hline \hline & Sup LM & exp LM & Double Max \\
\hline Test statistic & $403.93^{* *}$ & $195.78^{* *}$ & $7.89^{* *}$ \\
Breaks 1990, 1998 & before 1990 & $1990-1998$ & after 1998 \\
Mean of 2nd BI & $40.88^{* * *}$ & $32.87^{* * *}$ & $68.21^{* * *}$ \\
Std. error & 0.99 & 1.54 & 1.2 \\
\hline
\end{tabular}

* Significant at $5 \%$

** significant at $1 \%$

*** Significant at $0.1 \%$

while the process for the variance remains stable. The process for the mean have two peaks, the first one is about 1990 and the second one is in 1998. Again by estimating the break points the changes of the 2nd birth interval means correspond to break years are presented in Table 5 .

Table 5 shows that the 2nd birth interval first increased in 1990 (end of war), then decreased between (1990-1998) and strongly increased in recent years (from 1998 until 2002) against having trivial influence on the 1st birth intervals. The socio-economic and family planning factors have strong roles on the 2nd birth spacing.

The same as previous section a key question for our analysis is whether or not any effect of the socio-economic and cultural variables on the 2nd birth intervals remain unchanged over the time of couple's marriage?

Figure 4 shows the empirical process of Double max test considering linear effects of the socio-economic and cultural variables on the 2nd birth interval which exceeds $5 \%$ critical value (solid line). Noting that in Figure 3 stability of the 2 nd birth interval process has been tested without any factors effect. Hence there is evidence for structural change and it is clear that there are two shifts one about 1983 and the other one about 1998 (It should be emphasized that other test statistics lead to the same results which are not reported here). The Bayesian Information Criteria (BIC) selects the model with two breaks which confirm the result of previous test (Figure 5).

Figure 6 reveals the factors' shift during the time of couples' marriage. As we can see women's age at the 1st marriage, parents planning for the number of ideal children, the 1st birth interval and duration of the 1st breastfeeding exhibit structural change over the time of couple's marriage as the corresponding processes cross their boundary, while other factors show some fluctuation but none of them are significant. Thus the structure of the 2nd birth interval has changed over the time of couples' marriage. The estimated 


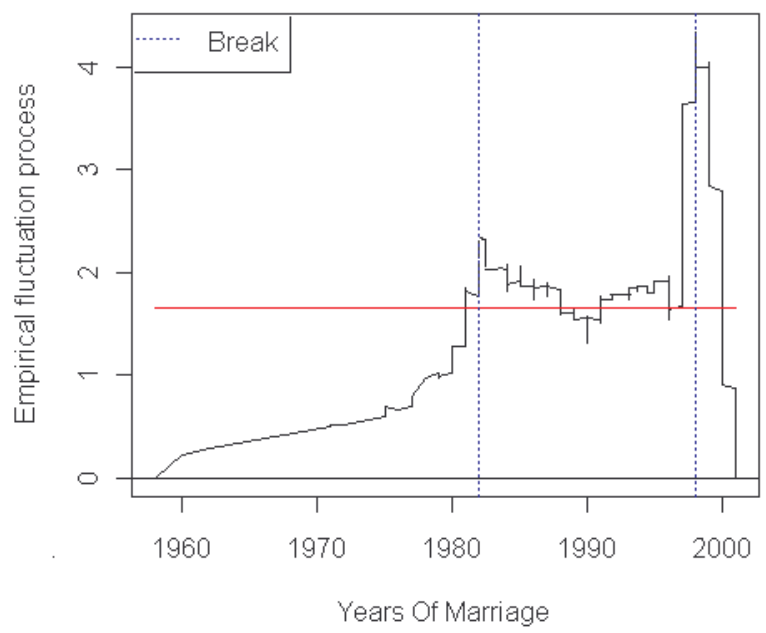

Figure 4. M-fluctuation process for the 2 nd birth interval with socio-economic effects

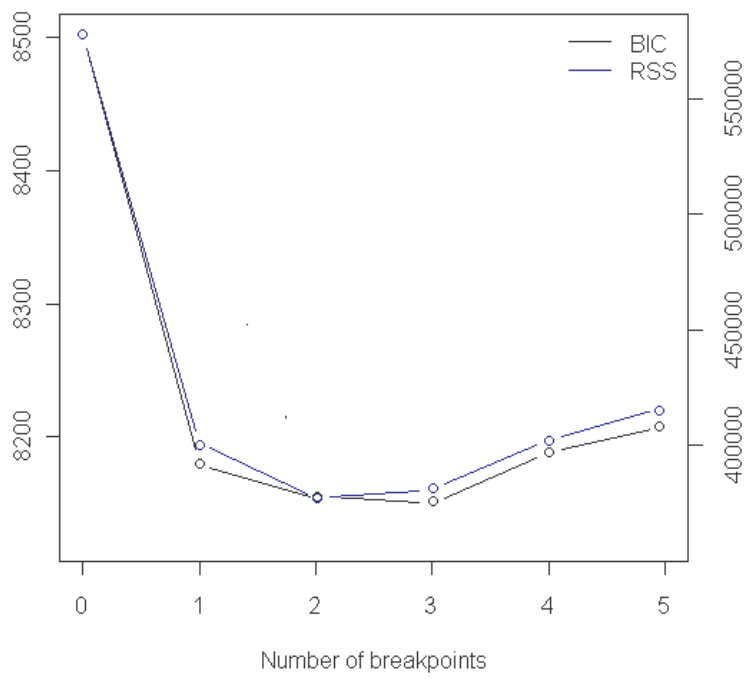

Figure 5. RSS and BIC for the 2st birth intervals 
break points imply that the 1st break occurred in 1989 which is after the Iranian revolution and beginning the Iraq-Iran war and the second break occurred in 1998.

Table 6 shows the segmented estimate of unstable factors effect on the 2nd birth interval during the years of couple's marriage. From this table we find out that parents' view about the ideal number of children has more opposite effect on the 2nd birth interval between (1989-1998) as compared to other years.
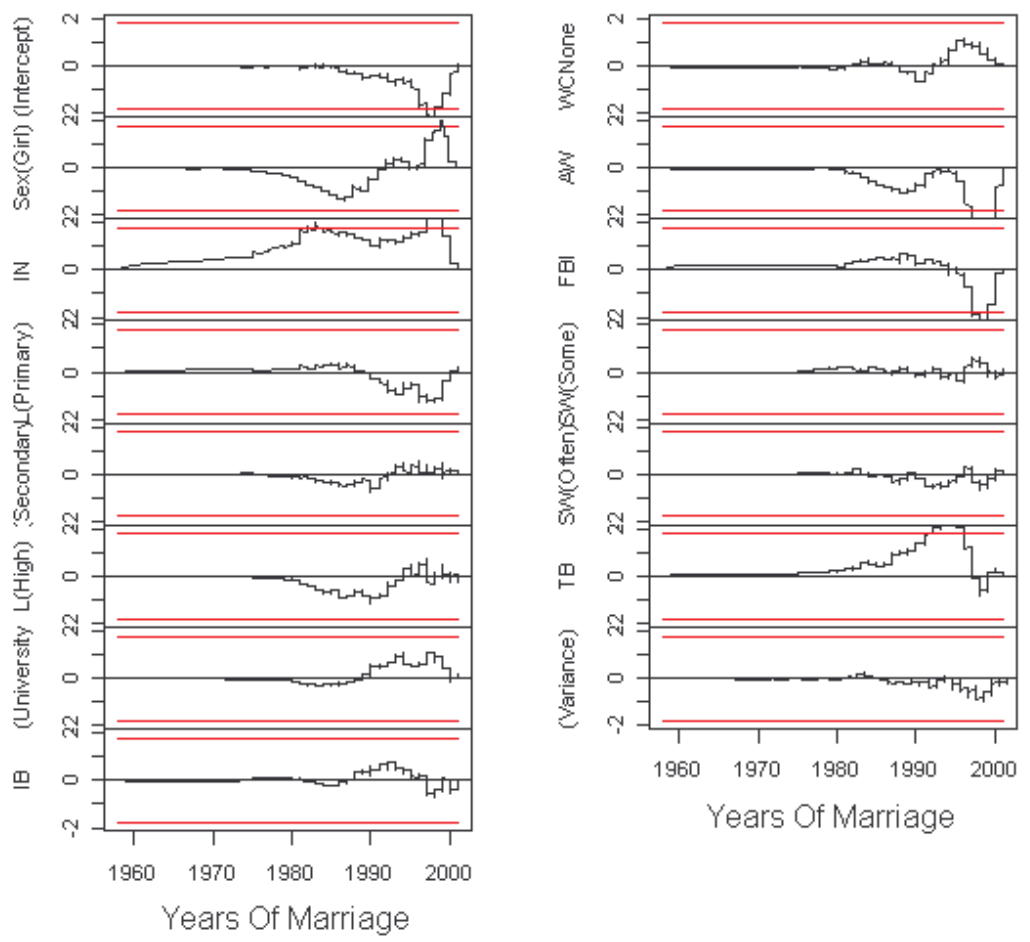

Figure 6. 13-dimensional empirical M-fluctuation process for the 2nd birth interval data with double maximum functional 
Table 6. Estimate of change effect of factors during years of marriage (standard error)

\begin{tabular}{lccc}
\hline \hline Variable & before 1989 & $1989-1998$ & after 1998 \\
\hline Ideal number of children & $-3.91^{*}$ & $-5.31^{* *}$ & $-2.83^{*}$ \\
Women's age at 1st marriage & $-0.61^{*}$ & -0.30 & -0.21 \\
Duration of breastfeeding & $0.58^{*}$ & $0.46^{*}$ & 0.15 \\
Sex (female) & $-4.21^{*}$ & -2.71 & $3.69^{*}$ \\
\hline
\end{tabular}

*Significant at $5 \%$

** significant at $1 \%$

The opposite effect of women's age at the 1st marriage on the 2nd birth interval decreased over the time of marriage. Duration of breastfeeding in last years of marriage has significantly more direct effect on the 2nd birth interval, while the effect of this variable decreased and is less significant in recent years. The influence of the sex of the 1st child on the length of the 2nd birth interval has changed over time. It can be seen that the opposite influence of female sex on the 2nd birth interval decreased during the time of marriage in such manner that after 1998 had direct effect on the 2nd birth interval.

Finally, Table 7 shows estimated effects of significant socio-economic and cultural factors on the 2nd birth interval from the whole data set (again we entered all available variables in the model but we report only the results of some of the variables including significant variables). The aim of this table is to compare the difference between estimated effects of factors from the whole data with those from the segmented data. The simple linear effects in this table show that by increasing the ideal number of children and women's age at the 1st marriage, the 2 nd birth interval decrease. However, the size of decrease in tables 6 and 7 are different.

It is known that breastfeeding reduces the risk of conception in women, so by increasing duration of breastfeeding the length of the 2 nd birth interval will be increased. However, from Table 7, the duration of breastfeeding in Table 7 has reciprocal effect on the 2 nd birth interval, while from Table 6 it is obvious that this factor has direct effect on the 2 nd birth interval. From Table 7 if the first child was female the 2 nd birth interval would be longer than if the 1st child was male, while a different result is derived from Table 6. Looking at educational level variable, it is clear the women with high education have more influence on the 2 nd birth spacing rather than other levels of education. 
80 Instability of the Determinants of Birth Intervals in Ahvaz-Iran: A Structural ...

Table 7. Estimated effect of socio economic factor on the 2st birth interval

\begin{tabular}{lcc}
\hline \hline Variable & Est. & Std. Error \\
\hline Constant & 55.87 & 6.11 \\
Sex & & \\
$\quad$ Female & $3.56^{*}$ & 1.42 \\
$\quad$ Male & - & - \\
Ideal number of children (IN) & $-6.26^{* *}$ & 1.59 \\
Women's view about ideal birth intervals (IB) & $0.88^{*}$ & 0.38 \\
Duration of 1st breastfeeding (TB) & -0.11 & 0.11 \\
Women's age at 1stmarriage (AW) & $-0.55^{*}$ & 0.22 \\
1st birth interval (FBI) & $-0.14^{*}$ & 0.06 \\
Wanting 2nd child & & \\
$\quad$ Both & - & - \\
$\quad$ None & $-10.26^{* *}$ & 2.177 \\
Women's level of Education & & \\
$\quad$ College & - & - \\
$\quad$ Primary & 0.43 & 3.59 \\
$\quad$ Secondary & 4.49 & 3.68 \\
$\quad$ Highschool & $7.63^{*}$ & 3.65 \\
$\quad$ None & $-12.33^{* *}$ & 4.05 \\
Women's access to media & & \\
$\quad$ Seldom & - & - \\
$\quad$ Sometimes & 2.03 & 1.73 \\
Always & $4.22^{*}$ & 1.92 \\
\hline
\end{tabular}

*Significant at $5 \%$

** significant at $1 \%$

*** Significant at $0.1 \%$

\section{Conclusion}

Structural change is an important issue in many areas of research and data analysis. Tests for structural change are valuable tools both in diagnostic checking and in graphical exploration of the structure of the data. In this paper, we used structural change approach to study the changes of demographic, family's view and socio-economic linearity effects on birth intervals during the years of couple's marriage in Ahvaz-Iran. Moreover, we studied the fluctuation of birth intervals mean process over these years. For the 1st birth interval using M-fluctuation test, we found that there were structural change in the mean of birth intervals in 1998, such that the mean increased after 1998 compared to before, while the structure of the relationship be- 
tween the 1st birth interval and some of important determinant variables remain stable over the period of couples' marriage.

Considering the 2nd birth intervals, we realized the mean of the 2 nd birth interval changed twice over time, the first decrease starts about 1990 and continue to 1998 and the second one starts increasing from 1998. The decrease in the mean of birth intervals during 1990-1998 can be related to peaceful situation of society. The increase in the mean after 1998 can be interpreted as the couple's decision to have fewer children and considering more space between the births. The structure of relationship of the 2 nd birth interval with determinant variables was also examined and instable variables were identified as their effect on the 2nd birth interval changed over the period of couples' marriage.

The influence of family's view about the number of children decreased the length of the second birth in 1989-1998 more than the one for other years. Also addressing those in recent years, the effect of women's age on the 2nd birth interval decreased and the influence of duration of breastfeeding had not significant. The decreasing in the 2 nd birth interval by sex of first child before 1989 was more than other years. In other words during the years of couple's marriage the reverse influence of female birth on the 2nd birth interval reduced in such manner that after 1998 a female birth increased the second birth space. It can be concluded that in recent years the sex of first child was not very important for couples.

\section{Acknowledgement}

We wish to thank the Editor and the referees for their valuable comments which enhanced the presentation of this paper.

\section{References}

Abdel-Aziz, A. (1983). A study of birth intervals in Jordan. Scientific Report No. 46, Netherland: World Fertility Survey.

Amani, F., Kazemnejad, A., Habibi, R. and Hajizadeh, E. (2011). Change point in Poisson distribution with application in Iranian mortality rate data. Iranian journal of epidemiology, 7, 15-22.

Andreou, E. and Ghysels, E. (2002). Multiple breaks in financial market volatility dynamics, Journal of Applied Econometrics, 17, 579-600. 
Andrews, D.W.K. (1993). Tests for parameter instability and structural change with unknown change point, |textitEconometrica, 61, 821-856.

Andrews, D.W.K. and Ploberger, W. (1994), Optimal tests when a nuisance parameter is present only under the alternative, Econometrica, 62, 1383-1414.

Bai, J. and Perron, P. (2003). Computation and analysis of multiple structural change models, Journal of Applied Econometrics, 18, 1-22.

Brown, R.L., Durbin, J. and Evans, J.M. (1975). Techniques for testing the constancy of regression relationships over Time, Journal of the Royal Statistical Society B, 37, 149-163.

Bumpass, L., Rindfuss, R. and Hanosik, R.B. (1978). Age and marital status at first birth and the pace of subsequent fertility, Demography, 15, 75-86.

Cooper, S.J., Piehl, A.M., Braga, A.A. and Kennedy, D.M. (2001)."Testing for structural breaks in the evaluation of programs," Unpublished Paper, John F. Kennedy School of Government.

Hansen, B.E. (1997). Approximate asymptotic p-values for structural change tests, Journal of Business 85 Economic Statistics, 15, 60-67.

Hansen, B.E. (1992). Testing for parameter instability in linear models, Journal of Policy Modeling, 14, 517-533.

Hjort, N.L. and Koning, A. (2002). Tests for constancy of model parameters over time, Nonparametric Statistics, 14, 113-132.

Kuan, C.M. and Hornik, K. (1995). The generalized fluctuation test: A unifying view, Econometric Reviews, 14, 135-161.

Mazanec, J.A. and Strasser, H. (2000). A Nonparametric Approach to Perceptions-based Market Segmentation: Foundations, Springer, Berlin.

Muggeo, V.M.R. (2003). Estimating regression models with unknown break-points, Statistics in Medicine, 22, 3055-3071.

Nair, S.N. (1996). Determinants of birth intervals in Kerala: An application of Cox's hazard model, Genus LII, 47-65.

Nyblom J (1989). Testing for the constancy of parameters over time, Journal of the American Statistical Association, 84, 223-230.

Rasekh, A.R. and Momtaz, M. (2007). The determinants of birth intervals in Ahvaz-Iran: A graphical modeling approach, Journal of Data Science, 5, 555-576.

Rashid Manan, H. (1997). Effects of lactation, contraception and other factors on birth intervals in Bangladesh: Evidence from the 1989 BFS, Genus LIII, 145-157. 
Stock, J. and Watson, M. (1996). Evidence on structural instability in macroeconomic time series relations, Journal of Business and Economic Statistics, 14, 11-30.

Suwal, J.V. (2001). Social-cultural dynamics of birth intervals in Nepal, Contribution to Nepalese studies (CNAS) Journal, 28, 11-33.

Trussell, J., Martin, L.G., Feldman, R., Palmore, J.A., Concepcion, M. and Abu Bajer, D.N. (1985). Determinants of birth intervals length in the Philippines, Malaysia and Indonesia: A hazard model analysis, Demography, 22, 145-168.

West, K.K. (1987). Education, age at marriage or first birth and labour force participation as predictors of parity transition: an application of discrete multivariate analysis to the study of conditional life table probabilities, Canadian Studies in Population, 14, 89-109.

Zeileis, A. and Hornik, K. (2003). Generalized m-fluctuation tests for parameter instability, Report 80, SFB "Adaptive Information Systems and Modeling in Economics and Management Science".

Zeileis A., Leisch F., Hornik K. and Kleiber, C. (2002). Strucchange: An R package for testing for structural change in linear regression models, Journal of Statistical Software, 7, 1-38. URL http://www.jstatsoft.org/v07/i02/.

Yao, Y.C. (1988). Estimating the number of change points via Schwartz Criterion, Statistics and Probability Letters, 6, 181-189.

\author{
Abdolrahman Rasekh \\ Department of Statistics, \\ Shahid Chamran University, \\ Ahvaz, Iran. \\ email: rasekh_a@scu.ac.ir
}

\author{
Hadi Imami \\ Department of Statistics, \\ Shahid Chamran University, \\ Ahvaz, Iran. \\ email: hadi.imami@gmail.com
}

